\title{
12. LEG 4 GRAIN SIZE RESULTS
}

\section{A. C. Pimm, Deep Sea Drilling Project, East Coast Repository Lamont-Doherty Geological Observatory, Palisades, N. Y.}

\section{INTRODUCTION}

A complete list of the grain size results is given as Table 1; triangle diagrams for each hole are given as Figure 1; and, the sand/silt and silt/clay boundaries downhole are shown in Figure 2A, B. Methods used for grain size analysis are given in the appendix to this volume.

\section{RESULTS}

\section{Site 23}

A hole drilled at Site 23 mainly penetrated clays with a clay content of 85 to 90 per cent. The clays are olivegray in Cores 1 and 4, and are yellowish-brown and zeolitic in Core 3 and part of Core 4 . Grain size samples from Core 5 are of silty clay and sand, probably of turbidite origin; the wide separation of the sand/silt and silt/clay boundaries in different parts of the same core is seen in Figure 2.

\section{Site 24}

Sediments from Holes 24 and 24A are described elsewhere in this volume as deep water geosynclinal "flysch" deposits. Cores 1 and 2 from Hole 24 are of sandy clay and clayey sand of terrigenous origin. In Core 4 from the same hole the sediments are brown and greenish-gray to blue with a clay content of 90 to 95 per cent. Cores 1 and 2 from Hole 24A contain clays similar to those from Hole 24. Silty clay and sand-siltclay from Cores 3 and 4 are terrigenous derived graywackes.

\section{Site 25}

All samples taken from Holes 25 and $25 \mathrm{~A}$ are of foraminiferal chalk ooze. The grain size, dependent upon the amount of foraminifera present, is mainly clayey sand or sandy clay in Cores 1 and 2, and sand-silt-clay in Cores 3 and 4 .

TABLE 1

Grain Size Results, Leg 4

\begin{tabular}{lcccrrrl}
\hline Hole & Core & Section & $\begin{array}{c}\text { Interval Sampled } \\
(\mathrm{cm})\end{array}$ & $\begin{array}{c}\% \\
\text { Sand }\end{array}$ & $\begin{array}{c}\% \\
\text { Silt }\end{array}$ & $\begin{array}{c}\% \\
\text { Clay }\end{array}$ & Classification \\
\hline 23 & 1 & 1 & $73-74$ & 0.5 & 13.3 & 86.2 & Clay \\
23 & 1 & 2 & 4 & 0.9 & 13.8 & 85.3 & Clay \\
23 & 1 & 5 & 0 & 6.6 & 14.0 & 79.4 & Clay \\
23 & 3 & 2 & 16 & 0.2 & 8.4 & 91.4 & Clay \\
23 & 3 & 3 & 23 & 0.2 & 7.5 & 92.3 & Clay \\
23 & 4 & 1 & $10-12$ & 5.7 & 9.5 & 84.8 & Clay \\
23 & 4 & 3 & $40-42$ & 1.9 & 7.5 & 90.6 & Clay \\
23 & 4 & 4 & $65-67$ & 1.9 & 8.7 & 89.4 & Clay \\
23 & 5 & 1 & 22 & 17.8 & 21.0 & 61.2 & Silty clay \\
23 & 5 & 1 & 46 & 83.2 & 6.8 & 10.0 & Sand \\
24 & 1 & 1 & $26-28$ & 42.7 & 12.0 & 45.3 & Sandy clay \\
24 & 1 & 1 & 34 & 25.7 & 10.2 & 64.1 & Sandy clay \\
24 & 2 & 1 & $15-16$ & 71.7 & 12.1 & 16.2 & Clayey sand \\
24 & 4 & 2 & 13 & 0.1 & 5.5 & 94.4 & Clay \\
24 & 4 & 3 & 29 & 2.3 & 8.5 & 89.2 & Clay \\
\hline
\end{tabular}


TABLE 1 - Continued

\begin{tabular}{|c|c|c|c|c|c|c|c|}
\hline Hole & Core & Section & $\begin{array}{l}\text { Interval Sampled } \\
\qquad(\mathrm{cm})\end{array}$ & $\begin{array}{c}\% \\
\text { Sand }\end{array}$ & $\begin{array}{c}\% \\
\text { Silt }\end{array}$ & $\begin{array}{c}\% \\
\text { Clay }\end{array}$ & Classification \\
\hline 24 & 4 & 4 & 6 & 0.2 & 4.0 & 95.8 & Clay \\
\hline $24 \mathrm{~A}$ & 1 & 1 & $1-24$ & 0.3 & 17.2 & 82.5 & Clay \\
\hline $24 \mathrm{~A}$ & 2 & 1 & $1-27$ & 0.3 & 20.6 & 79.1 & Clay \\
\hline $24 \mathrm{~A}$ & 3 & 1 & 84 & 0.3 & 24.9 & 74.8 & Silty clay \\
\hline $24 \mathrm{~A}$ & 4 & 2 & 115 & 32.4 & 20.7 & 46.9 & Sand-silt-clay \\
\hline 25 & 1 & 0 & 10 & 27.8 & 17.0 & 55.2 & Sandy clay \\
\hline 25 & 1 & 1 & 26 & 50.3 & 11.7 & 38.0 & Clayey sand \\
\hline 25 & 1 & 1 & 110 & 31.2 & 15.5 & 53.3 & Sandy clay \\
\hline 25 & 1 & 2 & 30 & 43.8 & 13.6 & 42.6 & Clayey sand \\
\hline 25 & 1 & 2 & 110 & 31.5 & 16.0 & 52.5 & Sandy clay \\
\hline 25 & 1 & 4 & 39 & 52.7 & 5.3 & 42.0 & Clayey sand \\
\hline 25 & 1 & 4 & 110 & 55.3 & 11.1 & 33.6 & Clayey sand \\
\hline 25 & 1 & 5 & 30 & 54.5 & 12.1 & 33.4 & Clayey sand \\
\hline 25 & 1 & 5 & 130 & 33.5 & 20.1 & 46.4 & Sand-silt-clay \\
\hline 25 & 1 & 6 & 68 & 62.3 & 9.3 & 28.4 & Clayey sand \\
\hline 25 & 2 & 2 & 30 & 60.2 & 0.4 & 39.4 & Clayey sand \\
\hline 25 & 2 & 3 & 70 & 50.2 & 12.6 & 37.2 & Clayey sand \\
\hline 25 & 3 & 1 & 125 & 18.7 & 28.0 & 53.3 & Silty clay \\
\hline 25 & 3 & 2 & 38 & 38.0 & 22.6 & 39.4 & Sand-silt-clay \\
\hline 25 & 3 & 2 & 114 & 43.3 & 23.8 & 32.9 & Sand-silt-clay \\
\hline 25 & 3 & 3 & 75 & 43.0 & 21.2 & 35.8 & Sand-silt-clay \\
\hline 25 & 3 & 4 & 75 & 43.7 & 19.1 & 37.2 & Clayey sand \\
\hline 25 & 3 & 5 & 75 & 46.1 & 22.4 & 31.5 & Sand-silt-clay \\
\hline 25 & 3 & 6 & 75 & 44.3 & 20.0 & 35.7 & Sand-silt-clay \\
\hline 25 & 4 & 1 & 33 & 44.6 & 17.8 & 37.6 & Clayey sand \\
\hline 25 & 4 & 2 & 75 & 48.3 & 24.3 & 27.4 & Sand-silt-clay \\
\hline 25 & 4 & 3 & 75 & 48.8 & 25.1 & 26.1 & Sand-silt-clay \\
\hline 25 & 4 & 4 & 76 & 46.5 & 25.3 & 28.2 & Sand-silt-clay \\
\hline $25 \mathrm{~A}$ & 1 & 1 & 7 & 74.7 & 12.0 & 13.3 & Clayey sand \\
\hline $25 \mathrm{~A}$ & 1 & 1 & 87 & 50.0 & 24.3 & 25.7 & Sand-silt-clay \\
\hline $25 \mathrm{~A}$ & 1 & 2 & 75 & 53.1 & 25.2 & 21.7 & Sand-silt-clay \\
\hline 26 & 1 & 2 & 10 & 32.3 & 18.6 & 49.1 & Sandy-clay \\
\hline 26 & 1 & 2 & 90 & 0.1 & 18.5 & 81.4 & Clay \\
\hline 26 & 1 & 3 & 13 & 0.1 & 20.2 & 79.7 & Clay \\
\hline 26 & 1 & 3 & 90 & 1.1 & 22.0 & 76.9 & Clay \\
\hline 26 & 1 & 3 & 138 & 9.4 & 79.1 & 11.5 & Silt \\
\hline 26 & 2 & 1 & 124 & 0.1 & 22.2 & 77.7 & Clay \\
\hline 26 & 2 & 2 & 10 & 0.1 & 24.4 & 75.5 & Clay \\
\hline
\end{tabular}


TABLE 1 - Continued

\begin{tabular}{|c|c|c|c|c|c|c|c|}
\hline Hole & Core & Section & $\begin{array}{l}\text { Interval Sampled } \\
\quad(\mathrm{cm})\end{array}$ & $\begin{array}{c}\% \\
\text { Sand }\end{array}$ & $\begin{array}{r}\% \\
\text { Silt }\end{array}$ & $\begin{array}{c}\% \\
\text { Clay }\end{array}$ & Classification \\
\hline 26 & 2 & 2 & 95 & 0.0 & 22.7 & 77.3 & Clay \\
\hline 26 & 3 & 2 & 20 & 0.0 & 21.3 & 78.7 & Clay \\
\hline 26 & 3 & 2 & 100 & 0.0 & 23.5 & 76.5 & Clay \\
\hline 26 & 5 & 1 & 13 & 0.0 & 15.6 & 84.4 & Clay \\
\hline 26 & 5 & 1 & 87 & 0.1 & 17.0 & 82.9 & Clay \\
\hline 26 & 5 & 2 & 26 & 0.0 & 16.6 & 83.4 & Clay \\
\hline 26 & 5 & 2 & 90 & 0.0 & 18.0 & 82.0 & Clay \\
\hline 26 & 5 & 3 & 27 & 0.0 & 19.5 & 80.5 & Clay \\
\hline 26 & 5 & 3 & 96 & 0.0 & 30.0 & 70.0 & Silty clay \\
\hline 26 & 5 & 4 & 20 & 0.1 & 71.1 & 28.8 & Clayey silt \\
\hline 26 & 5 & 4 & 87 & 0.0 & 19.2 & 80.8 & Clay \\
\hline 27 & 1 & 1 & 13 & 0.1 & 4.3 & 95.6 & Clay \\
\hline 27 & 1 & 2 & 15 & 0.1 & 5.6 & 94.3 & Clay \\
\hline 27 & 1 & 6 & 73 & 0.3 & 7.4 & 92.3 & Clay \\
\hline 27 & 2 & 1 & 86 & 0.2 & 8.4 & 91.4 & Clay \\
\hline 27 & 2 & 2 & 13 & 0.2 & 7.6 & 92.2 & Clay \\
\hline 27 & 2 & 3 & $21-23$ & 0.9 & 7.0 & 92.1 & Clay \\
\hline 27 & 2 & 3 & $80-82$ & 0.1 & 6.3 & 93.6 & Clay \\
\hline 27 & 3 & 1 & 47 & 0.1 & 8.3 & 91.6 & Clay \\
\hline 27 & 3 & 2 & $16-17$ & 0.1 & 10.4 & 89.5 & Clay \\
\hline 27 & 4 & 1 & 35 & 0.1 & 5.2 & 94.7 & Clay \\
\hline 27 & 4 & 2 & 4 & 0.6 & 8.9 & 90.5 & Clay \\
\hline 27 & 4 & 3 & 16 & 0.4 & 6.5 & 93.1 & Clay \\
\hline 27 & 5 & 1 & 19 & 0.5 & 7.4 & 92.1 & Clay \\
\hline 27 & 5 & 2 & 39 & 0.3 & 20.8 & 78.9 & Clay \\
\hline 27 & 5 & 3 & 25 & 4.0 & 19.4 & 76.6 & Clay \\
\hline 27 & 5 & 4 & 33 & 0.0 & 14.7 & 85.3 & Clay \\
\hline 27 & 6 & 2 & $90-100$ & 0.6 & 8.9 & 90.5 & Clay \\
\hline 27 & 6 & 3 & $20-30$ & 1.3 & 7.3 & 91.4 & Clay \\
\hline $27 \mathrm{~A}$ & 1 & 1 & $29-31$ & 0.1 & 8.9 & 91.0 & Clay \\
\hline $27 \mathrm{~A}$ & 1 & 1 & $77-79$ & 0.1 & 9.0 & 90.9 & Clay \\
\hline $27 \mathrm{~A}$ & 1 & 4 & $15-17$ & 0.0 & 8.4 & 91.6 & Clay \\
\hline $27 \mathrm{~A}$ & 1 & 5 & 31 & 0.1 & 8.8 & 91.1 & Clay \\
\hline $27 \mathrm{~A}$ & 1 & 6 & 18 & 0.1 & 9.7 & 90.2 & Clay \\
\hline $27 \mathrm{~A}$ & 1 & 6 & 90 & 0.1 & 9.0 & 90.9 & Clay \\
\hline $27 \mathrm{~A}$ & 2 & 3 & 25 & 0.2 & 14.2 & 85.6 & Clay \\
\hline $27 \mathrm{~A}$ & 2 & 3 & 73 & 0.2 & 75.6 & 24.2 & Silt \\
\hline $27 \mathrm{~A}$ & 3 & 1 & 132 & 0.2 & 21.2 & 78.6 & Clay \\
\hline
\end{tabular}


TABLE 1 - Continued

\begin{tabular}{|c|c|c|c|c|c|c|c|}
\hline Hole & Core & Section & $\begin{array}{l}\text { Interval Sampled } \\
\qquad(\mathrm{cm})\end{array}$ & $\begin{array}{c}\% \\
\text { Sand }\end{array}$ & $\begin{array}{c}\% \\
\text { Silt }\end{array}$ & $\begin{array}{c}\% \\
\text { Clay }\end{array}$ & Classification \\
\hline $27 \mathrm{~A}$ & 3 & 2 & 22 & 0.0 & 77.8 & 22.2 & Silt \\
\hline $27 \mathrm{~A}$ & 3 & 3 & 30 & 0.0 & 9.4 & 90.6 & Clay \\
\hline $27 \mathrm{~A}$ & 4 & 1 & $34-36$ & 0.0 & 8.3 & 91.7 & Clay \\
\hline $27 \mathrm{~A}$ & 4 & 2 & 49 & 0.0 & 11.1 & 88.9 & Clay \\
\hline $27 \mathrm{~A}$ & 4 & 3 & 15 & 0.1 & 15.1 & 84.8 & Clay \\
\hline $27 \mathrm{~A}$ & 4 & 3 & 77 & 0.3 & 70.5 & 29.2 & Clayey silt \\
\hline $27 \mathrm{~A}$ & 4 & 4 & 33 & 0.1 & 75.2 & 24.7 & Silt \\
\hline $27 \mathrm{~A}$ & 4 & 5 & 35 & 0.1 & 5.1 & 94.8 & Clay \\
\hline $27 \mathrm{~A}$ & 4 & 6 & 34 & 0.1 & 12.8 & 87.1 & Clay \\
\hline $27 \mathrm{~A}$ & 5 & 1 & $32-34$ & 0.0 & 10.1 & 89.9 & Clay \\
\hline $27 \mathrm{~A}$ & 5 & 2 & 46 & 0.3 & 14.3 & 85.4 & Clay \\
\hline $27 \mathrm{~A}$ & 5 & 3 & 22 & 0.2 & 10.6 & 89.2 & Clay \\
\hline $27 \mathrm{~A}$ & 5 & 4 & 57 & 0.1 & 12.5 & 87.4 & Clay \\
\hline $27 \mathrm{~A}$ & 5 & 5 & 84 & 5.0 & 1.5 & 93.5 & Clay \\
\hline 28 & 2 & 2 & 10 & 0.7 & 21.6 & 77.7 & Clay \\
\hline 28 & 2 & 3 & 16 & 0.2 & 28.9 & 70.9 & Silty clay \\
\hline 28 & 2 & 4 & 18 & 0.1 & 13.1 & 86.8 & Clay \\
\hline 28 & 3 & 1 & 11 & 0.1 & 64.4 & 35.5 & Clayey silt \\
\hline 28 & 3 & 2 & 36 & 0.2 & 74.1 & 25.7 & Clayey silt \\
\hline 28 & 3 & 3 & $42-44$ & 0.9 & 62.9 & 36.2 & Clayey silt \\
\hline 28 & 3 & 5 & 11 & 0.1 & 59.4 & 40.5 & Clayey silt \\
\hline 28 & 3 & 6 & 24 & 0.1 & 58.6 & 41.3 & Clayey silt \\
\hline 29 & 1 & 2 & 20 & 12.7 & 40.8 & 46.5 & Silty clay \\
\hline 29 & 1 & 3 & 30 & 4.7 & 30.6 & 64.7 & Silty clay \\
\hline 29 & 2 & 1 & 52 & 8.6 & 42.8 & 48.6 & Silty clay \\
\hline 29 & 2 & 3 & 50 & 2.3 & 19.7 & 78.0 & Clay \\
\hline 29 & 3 & 1 & 63 & 2.0 & 18.3 & 79.7 & Clay \\
\hline 29 & 4 & 1 & $109-111$ & 1.3 & 16.8 & 81.9 & Clay \\
\hline 29 & 4 & 2 & 53 & 2.4 & 15.5 & $\cdot 82.1$ & Clay \\
\hline 29 & 4 & 3 & 48 & 2.4 & 12.8 & 84.8 & Clay \\
\hline 29 & 4 & 4 & $16-19$ & 0.3 & 14.2 & 85.5 & Clay \\
\hline 29 & 5 & 1 & 122 & 0.0 & 7.1 & 92.9 & Clay \\
\hline 29 & 6 & 1 & $105-107$ & 0.1 & 7.9 & 92.0 & Clay \\
\hline 29 & 7 & 1 & 137 & 0.8 & 49.4 & 49.8 & Silty clay \\
\hline 29 & 8 & 1 & 118 & 23.0 & 61.5 & 15.5 & Sandy silt \\
\hline 29 & 9 & 1 & $47-49$ & 16.3 & 56.8 & 26.9 & Clayey silt \\
\hline 29 & 9 & 2 & 48 & 17.2 & 67.4 & 15.4 & Sandy silt \\
\hline 29 & 9 & 3 & 18 & 16.7 & 59.4 & 23.9 & Clayey silt \\
\hline
\end{tabular}


TABLE 1 - Continued

\begin{tabular}{|c|c|c|c|c|c|c|c|}
\hline Hole & Core & Section & $\begin{array}{l}\text { Interval Sampled } \\
\qquad(\mathrm{cm})\end{array}$ & $\begin{array}{c}\% \\
\text { Sand }\end{array}$ & $\begin{array}{c}\% \\
\text { Silt }\end{array}$ & $\begin{array}{c}\% \\
\text { Clay }\end{array}$ & Classification \\
\hline 29 & 9 & 4 & 35 & 10.6 & 47.7 & 41.7 & Clayey silt \\
\hline 29 & 9 & 5 & 21 & 8.7 & 41.0 & 50.3 & Silty clay \\
\hline 29 & 9 & 6 & 33 & 8.6 & 56.0 & 35.4 & Clayey silt \\
\hline 29 & 10 & 1 & 61 & 16.7 & 42.2 & 41.1 & Clayey silt \\
\hline 29 & 10 & 2 & $39-41$ & 8.6 & 45.5 & 45.9 & Silty clay \\
\hline 29 & 10 & 3 & $29-31$ & 10.5 & 39.3 & 50.2 & Silty clay \\
\hline 29 & 15 & 6 & 45 & 14.4 & 42.2 & 43.4 & Silty clay \\
\hline 29 & 16 & 1 & 46 & 10.0 & 42.1 & 47.9 & Silty clay \\
\hline 29 & 16 & 2 & 33 & 16.2 & 41.7 & 42.1 & Silty clay \\
\hline 29 & 16 & 3 & 46 & 20.4 & 41.7 & 37.9 & Sand-silt-clay \\
\hline 29 & 16 & 4 & 38 & 10.5 & 39.4 & 50.1 & Silty clay \\
\hline 29 & 16 & 5 & 32 & 19.8 & 38.8 & 41.4 & Silty clay \\
\hline 29 & 16 & 6 & 33 & 15.7 & 41.2 & 43.1 & Silty clay \\
\hline 29 & 17 & 1 & 31 & 23.2 & 40.8 & 36.0 & Sand-silt-clay \\
\hline 29 & 17 & 2 & 32 & 19.3 & 49.5 & 31.2 & Clayey silt \\
\hline 29 & 17 & 3 & 31 & 21.1 & 59.3 & 19.6 & Sandy silt \\
\hline 29 & 17 & 4 & 32 & 24.4 & 40.8 & 34.8 & Sand-silt-clay \\
\hline 29 & 17 & 5 & 32 & 19.9 & 42.7 & 37.4 & Clayey silt \\
\hline 29 & 17 & 6 & 32 & 18.8 & 44.6 & 36.6 & Clayey silt \\
\hline 29 & 18 & 2 & 42 & 18.2 & 42.2 & 39.6 & Clayey silt \\
\hline $29 \mathrm{~A}$ & 2 & 1 & 40 & 0.2 & 9.6 & 90.2 & Clay \\
\hline $29 \mathrm{~A}$ & 4 & 1 & 60 & 0.1 & 19.0 & 80.9 & Clay \\
\hline $29 A$ & 5 & 1 & $56-58$ & 0.1 & 11.4 & 88.5 & Clay \\
\hline $29 B$ & 1 & 1 & 33 & 0.0 & 22.6 & 77.4 & Clay \\
\hline $29 B$ & 1 & 4 & 131 & 0.0 & 8.0 & 92.0 & Clay \\
\hline $29 \mathrm{~B}$ & 1 & 5 & 37 & 0.1 & 8.8 & 91.1 & Clay \\
\hline $29 B$ & 1 & 6 & 41 & 0.1 & 9.0 & 90.9 & Clay \\
\hline $29 B$ & 2 & 2 & 41 & 0.6 & 11.4 & 88.0 & Clay \\
\hline $29 B$ & 2 & 3 & 22 & 0.0 & 10.2 & 89.8 & Clay \\
\hline $29 \mathrm{~B}$ & 2 & 4 & 22 & 0.1 & 14.5 & 85.4 & Clay \\
\hline $29 \mathrm{~B}$ & 3 & 1 & 42 & 0.2 & 24.9 & 74.9 & Silty clay \\
\hline $29 \mathrm{~B}$ & 3 & 2 & 28 & 0.1 & 11.6 & 88.3 & Clay \\
\hline $29 B$ & 4 & 1 & 27 & 0.1 & 10.7 & 89.2 & Clay \\
\hline $29 B$ & 4 & 2 & 27 & 0.2 & 11.4 & 88.4 & Clay \\
\hline $29 B$ & 4 & 3 & 32 & 0.1 & 7.3 & 92.6 & Clay \\
\hline $29 \mathrm{~B}$ & 4 & 4 & 28 & 0.1 & 5.7 & 94.2 & Clay \\
\hline $29 B$ & 5 & 1 & 31 & 2.1 & 25.2 & 72.7 & Silty clay \\
\hline $29 \mathrm{~B}$ & 5 & 2 & 27 & 4.5 & 44.0 & 51.5 & Silty clay \\
\hline
\end{tabular}


TABLE 1 - Continued

\begin{tabular}{|c|c|c|c|c|c|c|c|}
\hline Hole & Core & Section & $\begin{array}{l}\text { Interval Sampled } \\
(\mathrm{cm})\end{array}$ & $\begin{array}{c}\% \\
\text { Sand }\end{array}$ & $\begin{array}{c}\% \\
\text { Silt }\end{array}$ & $\begin{array}{c}\% \\
\text { Clay }\end{array}$ & Classification \\
\hline $29 \mathrm{~B}$ & 5 & 3 & 31 & 3.2 & 43.6 & 53.2 & Silty clay \\
\hline $29 \mathrm{~B}$ & 5 & 4 & 27 & 0.1 & 23.0 & 76.9 & Clay \\
\hline $29 \mathrm{~B}$ & 6 & 1 & 104 & 15.5 & 45.3 & 39.2 & Clayey silt \\
\hline $29 \mathrm{~B}$ & 8 & 1 & 31 & 21.5 & 43.3 & 35.2 & Sand-silt-clay \\
\hline $29 \mathrm{~B}$ & 8 & 3 & 26 & 24.8 & 47.9 & 27.3 & Sand-silt-clay \\
\hline $29 \mathrm{~B}$ & 8 & 4 & 31 & 24.0 & 50.9 & 25.1 & Sand-silt-clay \\
\hline $29 \mathrm{~B}$ & 8 & 5 & 31 & 27.7 & 48.1 & 24.2 & Sand-silt-clay \\
\hline $29 \mathrm{~B}$ & 8 & 6 & 26 & 25.8 & 47.2 & 27.0 & Sand-silt-clay \\
\hline $29 B$ & 9 & 1 & 36 & 20.7 & 38.4 & 40.9 & Sand-silt-clay \\
\hline $29 \mathrm{~B}$ & 9 & 2 & 28 & 17.1 & 37.5 & 45.4 & Silty clay \\
\hline $29 \mathrm{~B}$ & 9 & 3 & 27 & 16.9 & 13.7 & 69.4 & Sandy clay \\
\hline $29 \mathrm{~B}$ & 9 & 4 & 26 & 18.5 & 33.8 & 47.7 & Silty clay \\
\hline $29 \mathrm{~B}$ & 9 & 5 & 20 & 17.3 & 34.1 & 48.6 & Silty clay \\
\hline $29 \mathrm{~B}$ & 9 & 6 & 22 & 16.6 & 41.6 & 41.8 & Silty clay \\
\hline $29 \mathrm{~B}$ & 10 & 2 & 27 & 16.1 & 37.6 & 46.3 & Silty clay \\
\hline $29 \mathrm{~B}$ & 10 & 3 & 32 & 20.6 & 37.7 & 41.7 & Sand-silt-clay \\
\hline $29 \mathrm{~B}$ & 10 & 4 & 33 & 18.3 & 42.9 & 38.8 & Clayey silt \\
\hline $29 \mathrm{~B}$ & 10 & 5 & 44 & 19.1 & 43.6 & 37.3 & Clayey silt \\
\hline 30 & 1 & 1 & 115 & 14.4 & 18.6 & 67.0 & Silty clay \\
\hline 30 & 1 & 5 & 36 & 8.2 & 20.9 & 70.9 & Silty clay \\
\hline 30 & 2 & 3 & 31 & 9.8 & 23.7 & 66.5 & Silty clay \\
\hline 30 & 2 & 4 & 32 & 1.1 & 20.5 & 78.4 & Clay \\
\hline 30 & 3 & 2 & 31 & 2.2 & 25.2 & 72.6 & Silty clay \\
\hline 30 & 3 & 3 & 21 & 6.1 & 51.2 & 42.7 & Clayey silt \\
\hline 30 & 3 & 4 & 22 & 0.8 & 37.1 & 62.1 & Silty clay \\
\hline 30 & 4 & 1 & 110 & 7.2 & 36.5 & 56.3 & Silty clay \\
\hline 30 & 4 & 2 & 22 & 0.8 & 33.3 & 65.9 & Silty clay \\
\hline 30 & 5 & 1 & 42 & 2.2 & 16.2 & 81.6 & Clay \\
\hline 30 & 5 & 2 & 29 & 3.0 & 23.2 & 73.8 & Silty clay \\
\hline 30 & 6 & 1 & 31 & 1.3 & 23.4 & 75.3 & Clay \\
\hline 30 & 6 & 3 & 21 & 1.4 & 21.1 & 77.5 & Clay \\
\hline 30 & 7 & 1 & 68 & 2.0 & 19.6 & 78.4 & Clay \\
\hline 30 & 9 & 1 & 82 & 4.3 & 50.0 & 45.7 & Clayey silt \\
\hline 30 & 9 & 2 & 38 & 6.4 & 54.7 & 38.9 & Clayey silt \\
\hline 30 & 10 & 1 & 30 & 14.3 & 51.8 & 33.9 & Clayey silt \\
\hline 30 & 10 & 2 & 30 & 10.9 & 52.9 & 36.2 & Clayey silt \\
\hline 30 & 10 & 3 & 30 & 24.6 & 40.2 & 35.2 & Sand-silt-clay \\
\hline 30 & 11 & 2 & 25 & 2.3 & 54.8 & 42.9 & Clayey silt \\
\hline
\end{tabular}


TABLE 1 - Continued

\begin{tabular}{|c|c|c|c|c|c|c|c|}
\hline Hole & Core & Section & $\begin{array}{l}\text { Interval Sampled } \\
\qquad(\mathrm{cm})\end{array}$ & $\begin{array}{c}\% \\
\text { Sand }\end{array}$ & $\begin{array}{l}\% \\
\text { Silt }\end{array}$ & $\begin{array}{c}\% \\
\text { Clay }\end{array}$ & Classification \\
\hline 30 & 13 & 3 & 7 & 5.4 & 50.3 & 44.3 & Clayey silt \\
\hline 30 & 14 & 1 & 145 & 36.6 & 35.7 & 27.7 & Sand-silt-clay \\
\hline 30 & 15 & 1 & 136 & 32.5 & 39.4 & 28.1 & Sand-silt-clay \\
\hline 30 & 15 & 2 & 10 & 44.6 & 33.2 & 22.2 & Sand-silt-clay \\
\hline 30 & 15 & 3 & 10 & 41.4 & 38.1 & 20.5 & Sand-silt-clay \\
\hline 30 & 15 & 4 & 8 & 37.1 & 42.2 & 20.7 & Sand-silt-clay \\
\hline 30 & 15 & 5 & 7 & 27.8 & 50.5 & 21.7 & Sand-silt-clay \\
\hline 31 & 1 & 1 & 41 & 11.1 & 12.2 & 76.7 & Clay \\
\hline 31 & 1 & 2 & 30 & 9.2 & 8.8 & 82.0 & Clay \\
\hline 31 & 1 & 3 & 91 & 9.4 & 11.0 & 79.6 & Clay \\
\hline 31 & 1 & 4 & 30 & 10.3 & 10.9 & 78.8 & Clay \\
\hline 31 & 1 & 5 & 28 & 9.3 & 14.2 & 76.5 & Clay \\
\hline 31 & 3 & 1 & 25 & 10.5 & 14.9 & 74.6 & Silty clay \\
\hline 31 & 3 & 2 & 28 & 13.2 & 14.8 & 72.0 & Silty clay \\
\hline 31 & 3 & 3 & 36 & 8.7 & 22.3 & 69.0 & Silty clay \\
\hline 31 & 3 & 5 & 26 & 6.2 & 19.7 & 74.1 & Silty clay \\
\hline 31 & 3 & 6 & 31 & 11.9 & 5.2 & 82.9 & Clay \\
\hline 31 & 4 & 1 & 78 & 11.4 & 19.2 & 69.4 & Silty clay \\
\hline 31 & 4 & 2 & 39 & 15.0 & 18.3 & 66.7 & Silty clay \\
\hline 31 & 5 & 1 & 45 & 6.4 & 19.9 & 73.7 & Silty clay \\
\hline 31 & 6 & 1 & 32 & 4.2 & 18.6 & 77.2 & Clay \\
\hline 31 & 6 & 2 & 31 & 6.5 & 19.8 & 73.7 & Silty clay \\
\hline 31 & 7 & 1 & 65 & 4.7 & 20.2 & 75.1 & Clay \\
\hline 31 & 7 & 1 & 140 & 0.2 & 8.8 & 91.0 & Clay \\
\hline 31 & 8 & 1 & 58 & 1.3 & 20.2 & 78.5 & Clay \\
\hline 31 & 8 & 2 & 27 & 0.7 & 20.3 & 79.0 & Clay \\
\hline 31 & 9 & 2 & 58 & 9.0 & 43.8 & 47.2 & Silty clay \\
\hline 31 & 10 & 1 & $146-147$ & 1.8 & 51.4 & 46.8 & Clayey silt \\
\hline 31 & 10 & 2 & 143 & 0.6 & 50.2 & 49.2 & Clayey silt \\
\hline 31 & 10 & 3 & $146-148$ & 0.9 & 54.1 & 45.0 & Clayey silt \\
\hline 31 & 10 & 4 & 145 & 0.3 & 44.3 & 55.4 & Silty clay \\
\hline 31 & 10 & 5 & 145 & 0.3 & 50.0 & 49.7 & Clayey silt \\
\hline
\end{tabular}



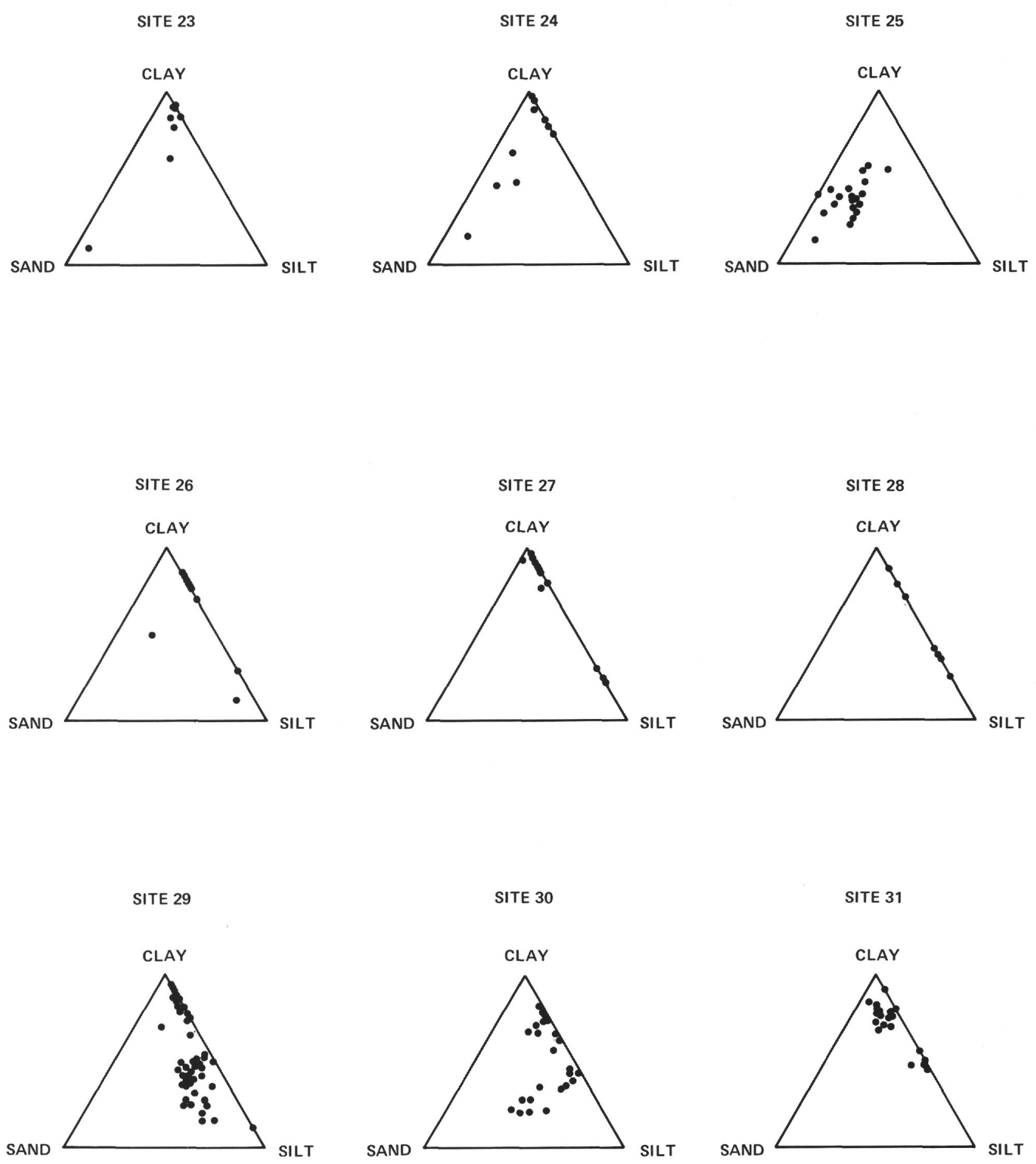

Figure 1. Triangle diagram of grain size from Leg 4. 


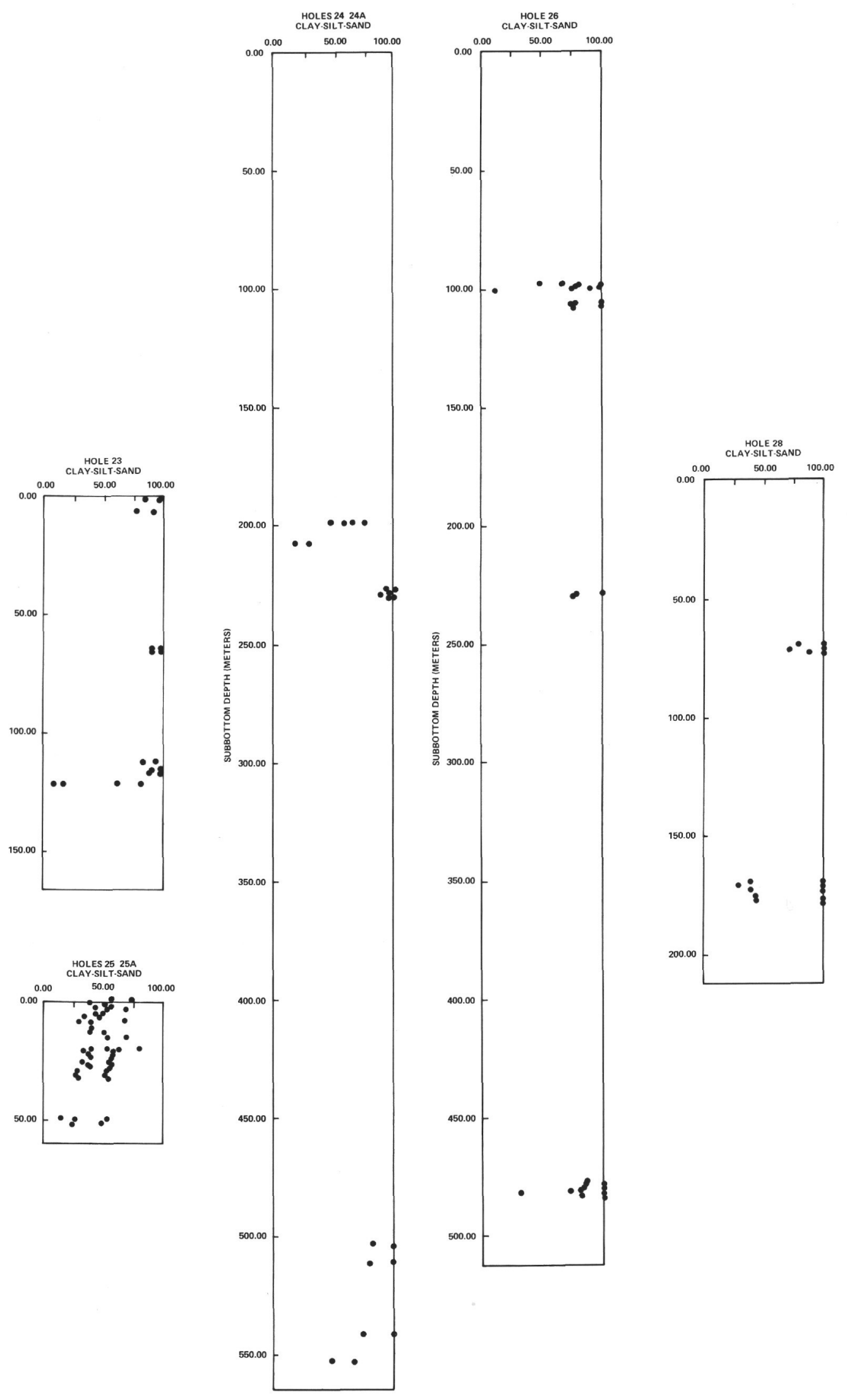

Figure 2A. Down-hole plots of sand/silt and silt/clay boundaries. 


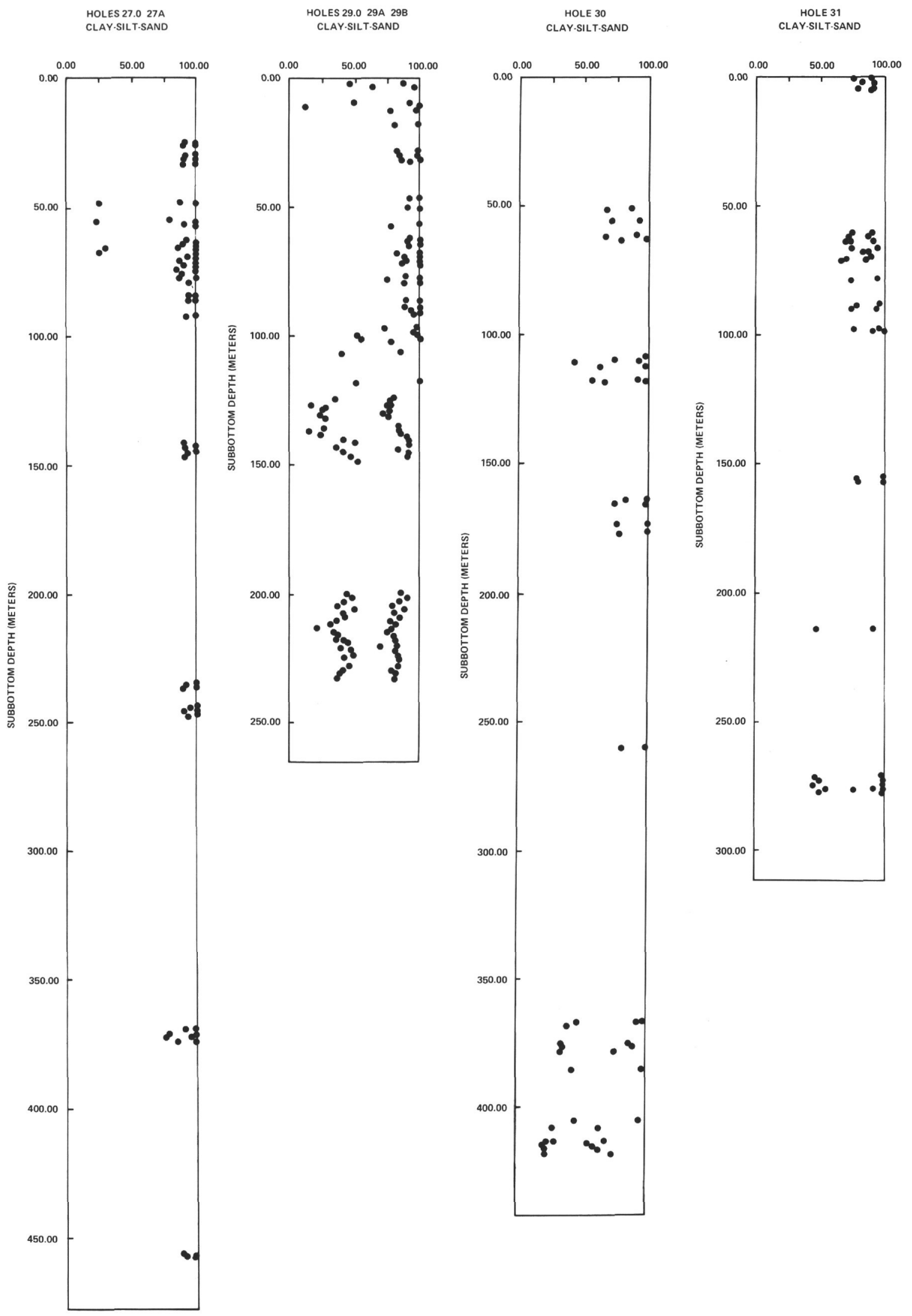

Figure 2B. Down-hole plots of sand/silt and silt/clay boundaries. 


\section{Site 26}

Sediments penetrated in this site are mainly olive-gray to medium gray clays containing about 75 to 85 per cent clay and 15 to 26 per cent silt. A few silt layers probably of turbidity current origin, occur in places (Core 1, Section 3). A graded bed ranging from clayey silt at the base through silty clay to clay at the top is seen in Sections 3 and 4 of Core 5. The presence of these coarser layers of sediment are particularly noticeable in Figure 2.

The lithological summary describes several fine to medium, and occasionally coarse sand stringers (of turbidite origin); but, these were either too thin for sampling, or just were not selected for grain size analysis.

\section{Site 27}

Hole 27 penetrated brown clay, in places greenish or grayish, with a clay content of $90+$ per cent.

Hole 27A contained grayish-brown, or rarely greenishbrown, clays with a clay content of 85 to 90 per cent. A few graded beds of silt and clayey silt occur in the clay; their occurrence is well exhibited in Figure 2. It should be noted that no nannoplankton were detected in sediments from this hole, and the silt fraction is made up of quartz, pyrite and manganese. In most other holes from Leg 4, and the other Atlantic sites, calcareous nannoplankton usually make up a sizeable proportion of the silt fraction (provided the sediment was deposited above the carbonate compensation depth).

\section{Site 28}

Sediments penetrated in this site were brown clay with thin silty clay layers in Core 2 , overlying clayey silt in Core 3 . The clayey silt contains 58 to 74 per cent silt, and less than 1 per cent sand, which is made up of nannoplankton and siliceous fossils.

\section{Site 29}

Hole 29 penetrated a variety of sediments, mainly of clay and silty clay size in the upper part of the section, but becoming coarser lower down due to a predominance of Radiolaria as the main fossil type, in contrast to nannoplankton in the upper part. Core 1 is a foraminiferal-nannoplankton chalk ooze of silty clay-size; Core 2 is similar but in places contains more or less nannoplankton and, therefore, the sediment either falls in the silt or clay size range. Cores 3 through 6 contain mainly olive brown and brown clays. Core 7 is a nannoplankton chalk ooze of silty clay size. Core 8 shows the beginning of the influx of Radiolaria and also a volcanic component; the sediment is a radiolarian ashey ooze of sandy silt size. Cores 9 and 10 are clayey silt and silty clay radiolarian oozes. Cores 15 through 18 are also radiolarian oozes and fall in the sand-silt-clay, clayey silt, and silty clay size ranges.

Hole 29A penetrated brown clay with a clay content of 80 to 90 per cent. Hole 29B penetrated a sequence of brown clays (Cores 1 through 4), silty clays and clayey silts composed of nannoplankton chalk ooze, (Cores 5,6 and 7), and then from Core 8 down the sediments contain siliceous fossils in place of the calcareous ones above. Core 8 is a sand-silt-clay composed of an ashey radiolarian ooze, and Cores 9 and 10 were silty clay or clayey silt of similar composition; the sand content (larger Radiolaria mainly) varies from 15 to 25 per cent.

\section{Site 30}

This site penetrated clay or silty clay in its upper part (Cores 1 through 7) and clayey silt or sand-silt-clay lower down (Cores 9 through 15). The finer-grained sediments in the upper part are greenish-gray, calcareous (nannoplankton) and contain less than 10 per cent sand-sized material. The higher sand and silt content of sediments in the lower part of the section here is due to an abundance of volcanic ash, fossils and glauconite.

\section{Site 31}

This site penetrated fine-grained sediments ranging in size from clay to clayey silt, the variation in grain size is related to the calcareous fossils present in the sediments.

Core 1 comprised 75 to 80 per cent clay, with sand and silt in about equal amounts. Core 3 is either silty clay or clay with both nannoplankton and formainifera (in this chalk ooze); Cores 4 through 6 are similar to Core 3 with silt varying from 15 to 20 per cent of the sediment. Cores 7 and 8 contain marl ooze of clay size. Cores 9 and 10 are silty clay or clayey silt, with silt varying from 43 to 54 per cent in these nannoplankton chalk oozes; the sand usually makes up less than 1 per cent of the sediment. 